\title{
Beyond Combination: How Cosmic Consciousness Grounds Ordinary Experience
}

\author{
'Man is a stream whose source is hidden. Always our being is descending \\ into us from we know not whence'. \\ Ralph Waldo Emerson, 'The Over-Soul'
}

\begin{abstract}
The aim of this paper is twofold. First, our purpose is to propose and motivate a novel and scientifically informed variant of cosmopsychism, namely, the view that the experiences of ordinary subjects are ultimately grounded in an all-pervading cosmic consciousness. Second, we will demonstrate that this approach generates promising avenues for addressing familiar problems of phenomenal constitution. We use stochastic electrodynamics (SED) as the physical bedrock of our approach, supplementing it with key insights about the nature of consciousness long emphasized in eastern philosophy and other wisdom traditions. We proceed to show that our approach substantiates an intriguing way of thinking about the dynamical emergence of ordinary consciousness from cosmic consciousness, identifying the latter with the vacuum state of quantum field theory. Finally, we argue that the present approach is well suited to address problems of phenomenal constitution, in particular as they pertain to the qualities and structure of experience and to the generation of subjects.
\end{abstract}

KEYWORDS: combination problem, cosmopsychism, panpsychism, stochastic electrodynamics, zero-point field, quantum coherence

\section{Introduction}

The last three decades witnessed a surge of interest in consciousness as a scientific and philosophical problem of the first rank. Correlated with this trend is a growing sense of dissatisfaction with standard materialist approaches to the mind-body problem: in particular with the explanatory gap associated with the notion that phenomenal consciousness is a cosmic latecomer emerging from an 
antecedent background of insensate physical stuff. Complementary to this latter sentiment is the revival of the idea that consciousness is ontologically fundamental, that is, an irreducible feature of ultimate reality or perhaps even the very essence of ultimate reality itself.

One approach that holds consciousness to be ontologically basic and enjoys growing popularity among scientists and philosophers who are critical of orthodox materialism is panpsychism. The distinctive markers of panpsychism can be thought of along two orthogonal dimensions: one horizontal, the other vertical. Horizontally, there is the idea that consciousness is immanent throughout nature, namely, that all concrete things are, in one way or another, infused with the dwelling presence of consciousness (hence the 'pan' in panpsychism). Vertically, there is the assumption that consciousness goes 'all the way down', which is to say that even prebiotic microscopic entities-no matter how small or simple-are endowed with a modicum of experiential life.

It is important, however, to avoid certain misconceptions regarding panpsychism. One prevalent misinterpretation is that panpsychism implies that all things are conscious. This, however, does not follow, for both the horizontal and the vertical dimension of panpsychism are entirely consistent with the notion that many macro-scale objects (rocks and chairs are typical examples) are inanimate, containing microscopic pockets of consciousness in their midst but lacking a unified consciousness extending across their full scale. Another misapprehension consists in the assumption that panpsychism is exhausted by micropsychism, the view that all conscious experiences are ultimately grounded in micro-level conscious experience. Again, there is nothing in the immanence hypothesis or even in the idea that consciousness goes all the way down to exclude the converse doctrine of cosmopsychism, according to which the ultimate ground of creaturely conscious experience is a cosmic-level consciousness.

In the early days of the revived interest in panpsychism, around the turn of the millennium, proponents and opponents alike were almost exclusively interested in atomistic or thing-pluralist variants of the doctrine, namely, in theories whose basic metaphysical assumptions reflect the reductionist bottom-up approach of orthodox materialism, with the important qualification that the elementary building blocks of nature (which were typically identified with subatomic particles) are considered to be endowed with rudimentary flashes of experience (see Chalmers I996; Seager 1995; Strawson 2006). ${ }^{\text {I }}$ More sophisticated manifestations of consciousness were presumed to result from one or another kind of combinatory integration-a mental chemistry, so to speak (see Coleman 2012; Goff 2006).

Following others, we call this brand of panpsychism micropsychism. The term is due to Strawson (2006) who uses it to denote a position that is somewhat weaker than panpsychism since it requires only that some microscopic ultimates are experience involving. Others, however, use 'micropsychism' as a label for atomistic panpsychism (see Chalmers, forthcoming; Goff, forthcoming). We find the latter use intuitive and will follow it henceforth.

\footnotetext{
${ }^{\text {I }}$ A more recent exposition of Strawson's view suggests that it is consistent with a quantum-field-based type of cosmopsychism (see Strawson, forthcoming).
} 
More recently, however, there is a growing interest in a holistic, thing-monist, alternative that came to be known as cosmopsychism (see, for example, Jaskolla and Buck 20I2; Goff 20I7; Mathews 20II; Nagasawa and Wager 20I7; Shani 20I5). On this latter view it is the cosmos as whole, rather than its tiniest bits and pieces, that is presumed to be ontologically fundamental and the ultimate ground of macro-level consciousness. As such, the assumption is that there is a cosmic level of consciousness, and that it is this cosmic consciousness (rather than microscopic forms of consciousness) that serves as the ultimate bedrock substantiating the experiential lives of creatures like us. ${ }^{2}$

A major trigger for the rise of interest in cosmopsychism is the hope that a holistic, top-down, substantiation of macro-level experience will prove a more viable option than an atomistic, bottom-up constitution. Micropsychism experiences serious difficulties in facing the combination problem, and advocates of cosmopsychism belong with those who suspect that a sound solution requires a radical shift in perspective (but see Dainton [20II], Miller [20I8], and Roelofs [20I 5] for recent attempts to address the combination problem from a micropsychist perspective).

In essence, the combination problem (Seager I995) consists in the task of explaining how macro-level phenomenal consciousness-the ongoing flow of subjective experience with which we are personally acquainted and which we ascribe with confidence to other people and animals around us-results from the combination of fundamental micro-level experiences. Put differently and in broader terms, the challenge is to explain how fundamental micro-consciousness substantiates familiar macro-consciousness. It is generally agreed that the combination problem is the most pressing theoretical challenge facing panpsychism at the present and that the future of the panpsychist platform depends crucially on the ability to address this challenge.

However, it is by no means obvious that a cosmopsychist framework can deliver us from the difficulties of mental combination. In particular, since it appears that cosmopsychism is vulnerable to a decombination problem that mirrors the combination problem faced by micropsychism. For if macro-consciousness is grounded in cosmic consciousness, does this not require the assumption that macro-level experiences are dissected from cosmic level experiences in a process that reverses micropsychist combination? Why should top-down (or cosmic-macro) constitution be less problematic than bottom-up (micro-macro) constitution? Is not the very idea of mental constitution afflicted with insurmountable obstacles, from whichever angle it is approached? This is a serious and fair concern that cosmopsychists cannot ignore.

While it is not our goal to disprove micropsychism, we hold the opinion that the cosmopsychist framework presented below carries a potential for moving the discussion forward or, at the very least, for pointing in a promising direction. We have both defended a cosmopsychist approach to consciousness in the past-one

\footnotetext{
${ }^{2}$ Apart from micropsychism and cosmopsychism, there is also a body of work that falls under the banner of emergentive panpsychism (see Brüntrup 2017; Mørch 2014; Rosenberg 2004; Seager 2017). Although marked with holistic overtones, emergentive panpsychism does not imply the reality of cosmic consciousness, thereby constituting a third alternative. While we recognize the significance of this line of research, we shall not discuss it any further here.
} 
of us from a philosophical standpoint (Shani 20I 5 ), the other from the standpoint of modern physics (Keppler 20I2, 20I3, 20I6, 20I8). In the present paper we argue that a combined approach that integrates both lines of work yields the resources for addressing some of the major difficulties associated with cosmic-macro constitution and the decombination problem.

In the next section we comment on some of the major combination and decombination problems discussed and debated in the philosophical literature. This will serve to clarify the terrain and to highlight the explanatory challenges faced by cosmopsychism with regard to mental constitution. It will also set up the problem space we target in later sections. In section 2 we introduce stochastic electrodynamics (SED), a nonstandard approach to quantum theory and fundamental physical reality, as the conceptual framework that will serve as the physical basis of our approach. In section 3 we show how this conceptual framework sustains an explanation of the emergence of ordinary experience against the background of an all-pervading field of cosmic consciousness. In section 4 we return to issues of mental combination, showing how an SED-based cosmopsychism opens up a promising avenue for solving a variety of constitution problems pertaining to the structure, and the qualities, of subjective experience. In section 5, we tackle the subject combination problem, arguably the most formidable of all problems of mental constitution. Finally, in section 6 we address some additional questions pertaining both to the conceptual soundness of our proposal and to issues of philosophical import that go beyond the scope of the present paper.

\section{Micropsychism, Cosmopsychism, and the Challenge of Phenomenal Constitution}

As mentioned above, the most formidable challenge facing panpsychism is to explain the constitutive dependency of familiar macro-level consciousness upon fundamental consciousness. If panpsychists are right in their contention that in order to reintegrate consciousness in nature we must evoke the hypothesis that experience is elemental, then this hypothesis ought to prove itself explanatorily potent: in one way or another, primordial consciousness must ground the reality of evolved sophisticated phenomenologies. Yet, as William James ([I890] I950: vol. I, chap. 5) famously argued, it is difficult to conceive how one conscious mind may derive its existence, qua conscious mind, from another (or from a plurality of such minds). Each conscious mind, says James, is phenomenally self-contained, and this runs counter to the assumption of subjective overlap involved in the notion that the phenomenal life of any macro-level subject is literally composed of (or decomposed from) the phenomenal lives of other, more basic subjects.

One intuitive and prima facie attractive way of explaining the ontological dependency of macro-level consciousness upon fundamental consciousness is by reference to compositional constitution. On this view, experiences as well as conscious selves enter into relations of cross-level inclusion: they are literally composed of or fractured from other experiences and selves (microscopic or cosmic, as the case may be). Yet, the idea that experiences or experiencing selves 
enter into strict relations of combinatory inclusion breeds considerable difficulties. In the first place, in the spirit of James's critique, it is open to the objection that nothing seems to necessitate the formation of novel experiences or experiencing selves from compositional (or decompositional) operations upon preexistent experiences or selves. In the absence of such necessitation or entailment, the postulation of consciousness at the basis of things fails to guarantee the reality of macro-level consciousness-giving rise to an explanatory gap that appears analogous to the one afflicting orthodox materialism. ${ }^{3}$ Second, and worse still, there is the more radical contention that such putative inclusion relations are not merely explanatorily deficient or inconclusive, but altogether incoherent. Each conscious perspective, so goes the idea, is strictly exclusive of all others; hence, none can include or be included in another. Consequently, the price of insisting on interperspectival combination is exacted in the form of loss of mental coherence. (The subtleties of this question cannot be explored in full here; for more detailed discussions see Albahari [forthcoming]; Basile [20I0]; Coleman [20I4]; and Shani [2015]). The struggle to make good sense of phenomenal inclusion, and more generally of any relation of phenomenal grounding, is what gives the combination problem its bite. ${ }^{4}$

Let us now delineate in broad brush strokes the troubled territory of the combination problem in its various derivatives. For the sake of orderly exposition we shall focus first on a few conspicuous variants of the combination problem as they appear in the context of micropsychism before pointing to their presumed analogs within the problem space of cosmopsychism.

As mentioned earlier, the 'combination problem' is actually a genus term designating a family of related problems. Chalmers (2017) argues that the combination problem $(\mathrm{CP})$ can be broken down into three major subcategories (there are other problems, known in the literature, that do not fit neatly into this tripartite classification, but we will not elaborate on them here):

(I) The subject CP: How do micro-subjects combine to yield a macro-subject?

(2) The quality CP: How do micro-qualities combine to yield macro-qualities?

(3) The structure CP: How do microexperiential structures combine to yield macroexperiential structures?

Given the assumptions of compositional constitution and cross-level inclusion, the combination problem can be plotted against any of these three different axes. Consider first the subject CP, which many believe to be the most formidable of all combination problems (see, e.g., Coleman 20I4; Miller 20I8). Here the challenge

\footnotetext{
${ }^{3}$ Argumentation along this line often parallels familiar arguments directed against orthodox materialism: in particular in the form of conceivability arguments (see Chalmers 2017; Goff 2009), or knowledge arguments (Chalmers 2017).

${ }^{4}$ Interestingly, James's original statement of the problem (I 890: I62) can be interpreted as lending support to either of the two challenges discussed above: lack of necessitation and straightforward impossibility.
} 
is twofold. First, to explain why any collection of subjects, however arranged, should ever give rise to a higher, inclusive subject. Second, to demonstrate that the very idea of subjective inclusion-according to which macro-level conscious perspectives are literally constituted of myriads of copresent micro-perspectives-is coherent, involving no logical contradictions or epistemic absurdities (see above).

Moving to the quality $\mathrm{CP}$, the challenge here is to explain how the phenomenal richness of the world we know could possibly be accounted for in terms of the putatively austere phenomenal qualities manifested by, say, subatomic particles. The problem is particularly acute given a 'Russellian' panpsychist picture, according to which all basal phenomenal properties are realizers of (functionally characterized) primitive physical properties (see Chalmers 201 5; Lockwood I993; Smolin 2015). On such a picture, a meager palette of indistinct qualities is burdened with the task of combinatorially generating the splendorous and seemingly inexhaustible gamut of all possible experiences (this is the so-called palette problem, see Lockwood I993).

Finally, the structure CP consists in the alleged mismatch between the contours of everyday experience and the phenomenal structure we would naturally expect to result from combinatorial operations over microscopic experiences. Paradoxically, it appears that the structure of daily experience is both too rich and too poor when compared with the presumed structure of its microexperiential base. On the one hand, it is hard to fathom how the primitive structural properties of microscopic experiences could account for the considerable spatiotemporal and multimodal complexity of macroscopic experience. On the other hand, macro-level phenomenology appears remarkably coarse-grained when compared with the putative grainy structure of collections of microexperiences (this is the so-called grain problem, see Lockwood I993; Sellars I965).

Are these problems translatable, mutatis mutandis, to cosmopsychism? Many believe they are (see Chalmers, forthcoming; Miller 20I8). Of course, much depends on how we choose to characterize the cosmic consciousness that, on this view, substantiates all other experiencing beings (see sections 4 and 5 below). Nevertheless, it is easy to see how constitution problems analogous in form to those afflicting micropsychism can resurface in the context of cosmopsychism by (as it were) reversing the arrow of constitution.

A structure constitution problem arises when one considers how the structure of everyday experience might be grounded in the structure of cosmic experiences. This problem appears particularly pressing if one assumes that the cosmic consciousness at the basis of all things is phenomenally austere, namely, that its experiential landscape is barren and homogenous in comparison to the mental lives of evolved localized creatures like us (see Chalmers, forthcoming). The same assumption gives rise to a quality constitution problem: How can the qualitatively rich inner world of macro-subjects emerge from the meager phenomenal background afforded by cosmic consciousness? How can a barren cosmic landscape sustain multiple oases of experiential affluence in its midst?

However, as before, it appears that the hardest of all constitution problems pertains to the constitution of subjects. Analogously to the bottom-up formation of macro-subjects from micro-subjects, the top-down derivation of macro-subjects 
from a cosmic subject faces the challenges of necessitation and intelligibility. Concerning the first challenge, the question is in what sense the postulation of a cosmic consciousness as the ground of all things helps explain (let alone necessitate) the manifest reality of evolved localized subjects. Concerning the second, the challenge is to show that the ontic dependency of macro-subjects upon an overarching cosmic consciousness can be delineated coherently, without incurring epistemic absurdity.

On the face of it, then, all the generic constitution problems afflicting micropsychism can be reformulated as applicable to cosmopsychism. Nonetheless, we surmise that the turn toward cosmopsychism is more than a formal reversal of the arrow of explanation. It reflects a profound shift in metaphysical outlook and, as such, it brings novel conceptual resources to bear on the problems at hand. In particular, we believe that a cosmopsychist platform informed, on the one hand, by contemporary physics and, on the other hand, by rather ancient metaphysical assumptions about the ultimate nature of cosmic consciousness, effects radical changes in our approach to questions of phenomenal constitution. In addition, it can prove itself instrumental in our ability to confront these questions successfully. The rest of the paper is an attempt to substantiate this claim.

\section{SED as a Conceptual Foundation for Quantum Physics}

The physical bedrock of our approach is SED, the foundations of which were laid some fifty years ago (Marshall 1963, I965; Boyer 1969, 1975) and have been continuously advanced over the past decades (De la Peña-Auerbach and Cetto I977; De la Peña and Cetto I994, I995, 2001, 2006; De la Peña et al. 2009, 20I5). The primary goal of this theory consists in deriving the formalism of quantum mechanics and quantum electrodynamics (QED) from first principles. Crucially, SED is based on the conception that the universe is imbued with an all-pervasive electromagnetic background field, called zero-point field (ZPF). The undisturbed ZPF, which features unique properties, is a maximally disordered field, meaning that the field modes are completely uncorrelated among each other (De la Peña and Cetto I994, I995; De la Peña et al. 2009).

In principle, the background activity represented by the ZPF corresponds to the vacuum fluctuations of QED. Yet, there are significant differences. In the conventional interpretation of QED the vacuum field is viewed as an unpleasant ingredient of the theory that is degraded to a virtual field being solely responsible for small corrections on top of the quantum behavior of matter. By contrast, in the conceptual framework of SED the ZPF occupies center stage in that it is looked upon as the origin of the quantum behavior of matter (De la Peña et al. 20I5); this framework is further elucidated below. Before we get into the details, it should be pointed out that the universal background activity is not only composed of an electromagnetic field, but also of fields that mediate other fundamental forces, such as the weak and strong interaction. However, we will ignore the latter fields since our main emphasis lies on the treatment of physical systems that are dominated by the electromagnetic interaction, something that applies particularly to living matter. 
A Material system embedded in the ZPF Stochastic motion (irregular dynamics)
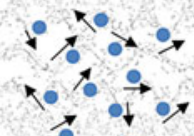

to

$\times$

C

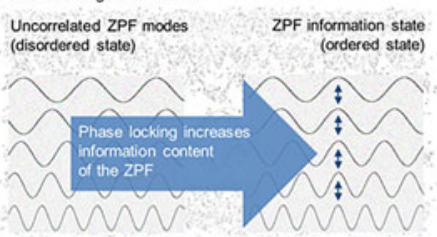

B

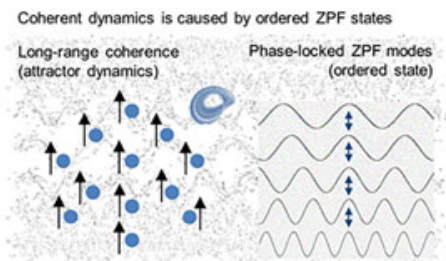

D sele

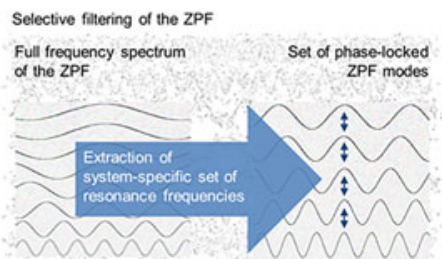

Figure I. (A) Every material system can be regarded as an open system in permanent contact with the random ZPF. Due to their interaction with the initially uncorrelated field modes, the system components acquire a stochastic motion and behave as stochastic oscillators. (B) A system that is sufficiently shielded from disruptive thermal influences can reach a dynamically stable state (i.e., an attractor) that is orchestrated by the ZPF. As a consequence, the system enters the quantum regime and displays long-range coherence, which in turn results in a phase-locked coupling of the field modes that are involved in the maintenance of the attractor dynamics. (C) Since such a phase locking increases the information content of the ZPF, the modified ZPF state accompanying the formation of an attractor can be viewed as a ZPF information state. (D) Seen from a different perspective, a quantum system features the characteristics of a resonant oscillator that extracts its system-specific set of resonance frequencies selectively from the full frequency spectrum of the background field.

According to SED, the electrically charged components of every physical system interact unavoidably with the radiative background (see figure IA), that is, every material system can be regarded as an open stochastic system in permanent contact with the random ZPF (De la Peña et al. 2015). As long as the interaction strength between the oscillating components and the relevant field modes, for which the system exhibits a strong resonant behavior, exceeds disturbing forces, such as thermal noise, the energy exchange between the system and the ZPF can reach equilibrium. In such a balance situation the ZPF takes control of the system and imposes restrictions on the dynamics of the system components that manifest themselves in quantization conditions in accordance with the stationary states predicted by quantum theory (De la Peña and Cetto I995, 200I, 2006). In other words, a system in equilibrium with the ZPF falls into a dynamically stable state, that is, an attractor, and displays quantum behavior (De la Peña and Cetto 1995).

Due to the close interrelationship between material systems and the background field, the presence of matter also exerts influence on the internal structure and dynamics of the ZPF. This interplay induces a modification and partial organization of the local field in such a way that the relevant ZPF modes, which can be regarded as a system-specific set of resonance frequencies playing a dominant role in the maintenance of the balance situation, become highly correlated (De la Peña and Cetto 2006; De la Peña et al. 2009). In other words, 
the orchestration of a dynamically stable system requires the initially chaotic ZPF to change over to a partially ordered state that shows a phase locking of the relevant field modes. As a result (see figure $\mathrm{IB}$ ), all the components of the system are effectively coupled through the ZPF, giving rise to collective cooperation and long-range coherence (De la Peña and Cetto 200I).

The causal relationships described above convey an idea of the fundamental mechanisms underlying quantum systems that can be interpreted with regard to both information gain in the ZPF and selective extraction of particular frequencies from the ZPF (Keppler 20I2, 20I3, 20I6). From the first perspective (see figure IC), the phase-locked ZPF modes that accompany the formation of an attractor represent a local ZPF information state that exhibits higher information content compared to the disordered initial state of the background field. Each attractor is characterized by its specific set of phase-locked field modes and, hence, by its unique ZPF information state. Seen from the second point of view (see figure ID), a system in equilibrium with the ZPF behaves as a resonant stochastic oscillator that extracts its resonance frequencies selectively from the full frequency spectrum of the background field. These system-specific ZPF modes undergo a phase-locked coupling while all the other modes remain unaffected.

Bearing this universal mechanism of structure formation in mind, it becomes obvious that there is no clear separation between the microcosm and the macrocosm, so that quantum behavior should not be restricted to the lowest levels of matter. The key insight is that coherent structures come into existence through selective filtering of the omnipresent $\mathrm{ZPF}$, in keeping with the guiding principle that the maintenance of the dynamic equilibrium in more complex material systems involves more complex ZPF information states. Correspondingly, quantum phenomena can be expected to appear in many macroscopic systems, particularly in living organisms, provided that they are sufficiently shielded from disruptive thermal influences (Del Giudice et al. 2005).

Beyond that, there is one more remarkable feature that can be attributed to the ZPF, namely, that not only the stability of matter, but also the putatively intrinsic properties of elementary particles, such as the quantized spin, turn out to be emergent phenomena arising from a deeper stochastic process involving the ZPF (De la Peña et al. 2009). This suggests that the ZPF can be seen as the root cause of the quantum behavior of matter and that all physical properties of matter can be understood as dynamically acquired properties resulting from the interaction with the background field.

In summary, SED paves a way for a deeper understanding and explanation of quantum phenomena and opens up new vistas that otherwise remain concealed behind the formalism of QED. In this way, it radically changes our notion of reality by giving significance to the ZPF as a creative agent that shapes matter and, due to its inherent stochasticity, constitutes the source of structural variety in the universe. In particular, the properties of any kind of quantum system can be traced back to the resonant interaction between the system components and the ZPF, which in turn modifies the background field and results in the formation of system-specific ZPF information states. In contrast, the components of classical systems are not dynamically coupled via the ZPF, thus leaving the ZPF completely 
A ZPF as the substrate of consciousness

All shades of phenomenal awareness are woven into the fabric of the ZPF.

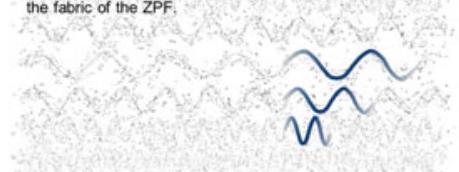

C

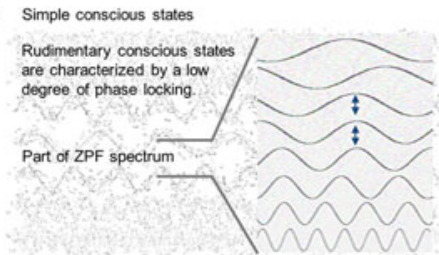

B ZPF information states are associated with conscious states

The universal mechanism behind conscious systems relies on the phase locking of ZPF modes.

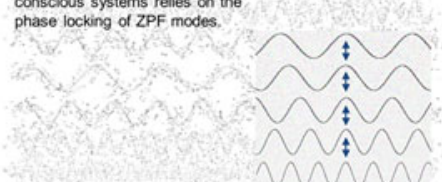

D Complex conscious states

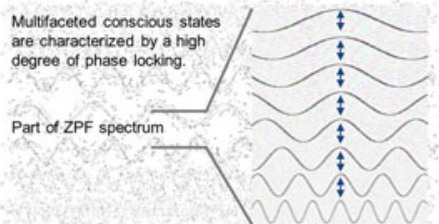

Figure 2. (A) The theoretical framework is based on the hypothesis that the all-pervasive ZPF is the carrier of consciousness, that is, all shades of phenomenal awareness are woven into the fabric of the ZPF. (B) Under this assumption, the principle of dynamical coupling of sets of ZPF modes is eminently suitable for the extraction of an enormous variety of shades of consciousness from the phenomenal color palette immanent in the ZPF. (C) The quantity of consciousness of a system is determined by the dynamically accessible part of the ZPF spectrum and the degree of phase locking the system is able to establish in this part. Simple conscious states are characterized by a low degree of phase locking. (D) In contrast, complex conscious states are characterized by a large number of phase-locked ZPF modes.

unaffected and rendering the system incapable of generating ZPF information states (Keppler 2016, 2018).

\section{SED as a Theoretical Framework for Consciousness}

The aforementioned features suggest that the ZPF is perfectly suited for playing the dual role as the carrier of both primordial energy and consciousness. Accordingly, we posit that all conceivable shades of phenomenal awareness are inherent in the frequency spectrum of the ZPF (see figure $2 \mathrm{~A}$ ). Due to its disordered ground state, the ZPF can therefore, from the external perspective, be regarded as a vibrant pool of activity, and from the internal perspective be looked upon as a formless sea of consciousness or unstructured ocean of awareness that carries an enormous range of potentially available phenomenal nuances. Proceeding from this postulate, the mechanism underlying quantum systems meets the requirements that are to be imposed on a truly fundamental mechanism behind conscious systems, leading us to the assumption that conscious systems extract their states of consciousness from the phenomenal color palette immanent in the ZPF (see figure $2 \mathrm{~B}$ ). These hypotheses express that every ZPF information state is associated with a conscious state or, put differently, that a pattern of phase-locked ZPF modes determines the physical as well as the phenomenal properties of a quantum system (Keppler 20I3, 20I6, 20I8).

As a consequence, it can be expected that every quantum system is a conscious system, with the accessible spectrum of conscious states of a given system being 
delimited by its dynamic variability, that is, by the variety of transiently stable attractors, and the quantity of consciousness of each state being determined by the degree of phase locking in the accessible part of the ZPF (see figures $2 \mathrm{C}$ and $2 \mathrm{D}$ ). These inferences are valid unless there are plausible additional constraints for the domain of consciousness, which we do not see at the moment. While simple quantum systems, such as atoms and molecules, are probably equipped with a very rudimentary, limited, and monotonous form of consciousness, we may assume that complex quantum systems, such as coherently oscillating cell assemblies in living organisms, are endowed with a broad range of multifaceted conscious experiences.

At this point, it is important to recall that the phenomenal properties of quantum systems are not intrinsic properties, but dynamically acquired properties that can be attributed to the system over the lifetime of an attractor. In contrast to quantum systems, the dynamics of classical systems are completely independent of the ZPF, thus leaving the background field unaffected and preventing the generation of ZPF information states. This suggests that such systems are excluded from conscious awareness (Keppler 2013, 2016, 2018) or, expressed differently, that it is nomologically impossible for classical systems to be conscious.

From this mechanism we obtain a clear demarcation criterion between conscious and nonconscious systems in such a way that the formation of transiently stable attractors distinguishing themselves by a high degree of coherence is an essential prerequisite for conscious processes; this is supported by an extensive body of evidence from neuroscience (Desmedt and Tomberg I994; Rodriguez et al. I999; Engel and Singer 200I; Melloni et al. 2007; Freeman 2007; Freeman and Vitiello 2007). Such attractors manage the transition from potentiality to actuality; that is, a potential conscious state is actualized once an attractor is fully unfolded and the concomitant ZPF information state is generated (Keppler 20I3, 20I8). In summary, our SED-based approach indicates that the creation principle in the universe, with respect to both physicality and phenomenology, is grounded in selective restriction of the omnipresent $\mathrm{ZPF}$.

\section{Reassessing Phenomenal Constitution in Light of the Filtering Hypothesis: Issues of Quality and Structure}

The framework presented in the last two sections bears conspicuously on matters of phenomenal constitution. This is particularly evident with regard to constitution problems pertaining to the quality and structure of experience. In essence, we believe that our approach circumvents this family of problems, making them largely irrelevant for the kind of cosmopsychism we advocate. The core issue at stake is the compositional assumption that the experiences of nonfundamental subjects derive their qualities and structure from the qualities and structure of experiences consciously entertained at the fundamental level of reality (by a plurality of subjects according to micropsychism or by a single subject if cosmopsychism is presupposed). In other words, the crucial presupposition behind common formulations of the quality and the structure combination problems is that the experiences of macro-subjects are literally composed of, or fractured from, 
manifest experiences of elemental subjects. As noticed above (see section I), the problem is to render these presupposed compositional relations intelligible. By contrast, the present framework breaks free from this core presupposition and in doing so avoids its tangles.

In the first place, our proposed framework avoids the assumption that there is a literal sense in which either the quality or the structure of the experiences of any single subject are derived (composed or fractured) from the manifest experiences of any other subject or subjects. Instead, as explained above, we employ the filtering hypothesis, according to which the phenomenal portrait of each quantum coherent system is a function of the manner in which it resonates with the ZPF, stirring the latter into a unique set of phase-locked modes. The individual dynamical properties and the contextual embeddedness of each such system ensure the uniqueness of the phenomenal portrait it carves for itself through its ongoing interaction with the ZPF, so that no two subjects are phenomenally identical in all respects. But insofar as the present problem is concerned, the main point is that no subject is phenomenally composed of or fractured from another subject; rather, each subject obtains its phenomenal character by tapping directly into the universal pool of cosmic consciousness immanent to the ZPF and by extracting from it a system-specific set of correlated resonance frequencies. (As panpsychists we still hold on to the idea that macro-subjects are physically constituted of micro-subjects: cells, atoms, etc. But the point is that once constituted as a complex physical entity with specific dynamical characteristics, each macro-subject obtains its phenomenology through resonant interaction with the background field, rather than by summing over the experiences of its micro-constituents.)

Equally important is the observation that while our approach identifies the conscious experiences of subjects with patterns of organization emergent within a universal field of cosmic consciousness (i.e., with phase-locked ZPF modes) we assume neither that these experiences are enjoyed by cosmic consciousness as its own nor that they constitute proper parts of extant cosmic experiences. Of crucial relevance, in this respect, is the distinction between implicit and explicit phenomenology. As mentioned earlier, we maintain that all conceivable shades of phenomenal consciousness are inherent in the frequency spectrum of the ZPF (see figure $2 \mathrm{~A}$ ). However, in the unorganized ground state of the background field these phenomenal nuances lie dormant and undifferentiated: they exist in potentia rather than in actualis, implicitly rather than explicitly. Thus, although the entire phenomenal 'color palette' is immanent in the ZPF, it would be a misinterpretation to conclude that a cosmic consciousness must therefore experience any of these potential states as an actual subjective experience: no ordinary concrete experience can be read into the ZPF in its default state of uncorrelated field modes.

Indeed, that our approach does not imply any ordinary phenomenal states on the part of cosmic consciousness can also be related from a different angle, that of traditional, spiritually based conceptions of cosmic consciousness. In line with such conceptions, we hold that cosmic consciousness is, fundamentally, a pure consciousness, that is, a formless sea of awareness that serves as the universal womb 
out of which all creaturely forms of experience arise. This conception of the ultimate ground of being as a pure consciousness is perhaps most articulately developed in Hinduism and Buddhism, but traces of it can be found in spiritual traditions the world over, including: Taoism; the mystery cults of the ancient world; Neoplatonism; Christian, Jewish, and Muslim mysticism; Aztec philosophy, and more.

Could this formless ground state be nevertheless endowed with some fundamental phenomenal attributes? In spiritual traditions as well as in reports based on deep states of meditation, pure consciousness is often associated with an ineffable sense of unconditioned bliss, love, and unity. But even assuming the reality of such cosmic phenomenology, insofar as the present discussion is concerned, the relevant point is that there is no viable sense in which our ordinary experiences can be thought of as bits and pieces dissected from it. In other words, no mereological assumption of phenomenal decombination is involved.

Finally, the confusion between implicit and explicit phenomenology bears upon another misconception concerning cosmopsychism. As mentioned in section I, a key assumption behind the quality and the structure decombination problems is the austerity bypothesis, which implies that if there is such a thing as cosmic consciousness, then its experiences are relatively shallow and homogenous. Thus, the problem is to explain how the allegedly meager phenomenal landscape of cosmic consciousness could possibly ground the lavish phenomenology of creatures like us. Again, the perspective we bring to the table avoids the problem because, as mentioned earlier, while we take the ZPF to be phenomenally indescribably rich, its richness is implicit and in potentia. Thus, we make no assumption to the effect that the universal background field from which our experiences are ultimately derived is phenomenally affluent in the same explicit manner in which human experience may be said to be so. Instead, our approach enables us to explain how the phenomenal character of our everyday experience is grounded in cosmic consciousness without violating the letter of the austerity hypothesis. ${ }^{5}$

\section{Cosmic Consciousness and the Constitution of Subjects}

We come at last to the subject constitution problem. As noted in section I, the challenge is to articulate a substantive and informative ontological sense in which the subjectivity of created subjects depends on the subjectivity inherent in cosmic consciousness-and to do so coherently, without succumbing to daunting conceptual aporia. The first task, to which we turn next, is to explain the place of subjectivity in the picture we advocate. Earlier, we pointed to the difference between cosmic consciousness and the consciousness of individual creatures by describing the former as pure consciousness, a limit state of consciousness characterized by a unitary sense of undifferentiated wholeness. In like manner

\footnotetext{
${ }^{5}$ Having said that, we consider the austerity hypothesis problematic in that it correlates informational richness with manifest physical complexity. While this assumption may be appropriate in classical physics, it does not seem in accord with quantum physics and the ontology of quantum fields. The ZPF is a case in point: judged by its surface structure it may appear barren and austere, but if one probes deeper into its potential for storing information and into the manner in which its dynamical properties substantiate all emergent concrete forms of matter, a very different perspective opens up.
} 
(and, again, in alignment with spiritually informed commentary on the nature of absolute consciousness) we maintain also that cosmic consciousness realizes an analogous limit state of subjectivity and can therefore be described as a pure subject.

A pure subject is an aperspectival subject, that is, a universal subject devoid of individual conscious perspective. Regular subjects experience their inner reality and the world around them in a conditioned manner constrained by the unique particularities of their creaturely being: their body physique; the qualities of their senses; their present situatedness; past experience and accumulated memory; drives, desires, and conscious purposes; established knowledge structures; attitudes and judgments; unconscious complexes and tendencies; language; social and cultural conditioning; etc. Such constraining factors serve to delimit and shape one's experiential flow: constituting a unique mode of opening to the world, a specific (albeit open-ended) angle through which things are experienced. This constrained and qualified opening to the world is what we understand by perspective (see Shani 20I5). It may be added also that the common manner in which perspectival subjects experience reality is dual: one experiences a world of objects (including inner objects, such as thoughts and feelings) 'over there', to be taken in by the here and now of one's own self. Thus, in contrast to regular subjects, a pure subject can be thought of as one whose subjectivity is free from the constraints of creaturely perspective and from the dual partitioning the latter imposes upon experience. In other words, the experience of a pure subject is aperspectival and nondual (for a detailed and informative analysis of this intriguing mode of subjectivity see Albahari, forthcoming).

Such negative characterization, however, gives rise to a legitimate concern, namely, whether a pure subject is a subject at all: in what sense can subjectivity survive the absence of perspective? What attributes, other than the existence of a perspective, could possibly justify the idea that cosmic consciousness possesses a subjective dimension? The answer to this question is that in the absence of perspective there remains ipseity, or selfhood as such: a conscious presence devoid of form and objects yet ready to assume ordinary qualitative tones and to serve as the apprehending recipient of objects if the right conditions for the emergence of an individual conscious perspective materialize. In the Hindu tradition this universal pure self is described as the Atman, and it is believed to be the ultimate ground of all perspectival conscious selves. ${ }^{6}$

Thus, on the view we advocate the ZPF is a bearer of pure consciousness as well as pure subjectivity. Consequently, consciousness and selfhood are posited as fundamental features of reality. What is not fundamental and therefore calls for explanation is the existence of perspectival selves endowed with structured

\footnotetext{
${ }^{6}$ In Advaita Vedanta and other spiritually informed wisdom traditions this conscious presence is often described as a witness-consciousness (see e.g., Fasching 20II). Albahari (forthcoming) associates witness consciousness with a present-moment sense of being, characterized by such properties as intransitivity (being nonobjectual) and reflexivity (being self-revealing). Such consciousness is also frequently described as luminous, by which it is meant that it possesses a power analogous to light, a power that 'illuminates or reveals things so they can be known' (Thompson 2015: 3). The concept of ipseity is regularly deployed in the phenomenological literature (e.g., Sokolowski 2000; Zahavi 2005), albeit without cosmic implications; but see Almaas (2004: ch. 2I) for a discussion of ipseity in relation to pure consciousness.
} 
experience. We have already demonstrated how structured experience could be intelligently grounded in the formless reservoir of cosmic consciousness. The remaining task is to explain how perspectival subjects emerge against the background of cosmic consciousness and in particular to explain how the subjectivity of such subjects is intelligently grounded in the pure subjectivity of the background field.

To do so, recall first what perspectival subjects are. They are localized centers of consciousness; they experience reality in a constrained and selective manner, through specific channels, portals, and filters (as it were); finally, although their field of experience is unified, it is structured and dually framed: presenting objects as given to an underlying apprehending recipient. Thus, if perspectival subjects are to appear on the cosmic scene, the minimal conditions for the materialization of these characteristic features must be met. We believe that the SED-based process dynamics described in sections 2 and 3 meets these minimal conditions. Let us first recapitulate the essentials of this process dynamics and then explain how it meets the prerequisites for the emergence of conscious perspectives.

In the first place, the physical narrative underlying our approach describes the formation of dynamically stable quantum systems in resonant equilibrium with the ZPF (see also Keppler 20I6). Moreover, as explained, the equilibrium conditions lead to a partial ordering of the local field, inducing long-range coherence in the emergent quantum regime (see figure IB, left). Finally, the same process whereby the ZPF is organized through the phase-locked coupling of the field modes involved in maintaining the attractor dynamics leads also to selective elicitation of structured phenomenal states (see figures $\mathrm{IB}$, right, and $2 \mathrm{~B}$ ). Each of these features, we argue, is relevant to the possibility of perspectival subjectivity.

First, the emergence of meta-stable attractors in energetic equilibrium with the ZPF marks the formation of localized regions of intensity-in constant interaction with, yet functionally distinct from, the surrounding field. Such intensified regions, which can also be thought of as vortices in the ocean of cosmic consciousness (Shani 2015), sustain an inner conscious domain that is shielded, to a degree, from its environment-a 'here' demarcated from whatever may lie out 'there'. Second, the physical boundaries of such stable organizations and the particularity of their individual characteristics serve as constraining factors that tether each system to specific modes of opening to the world, hence to a perspective. Third, the long-range coherence of such systems enables them (among other things) to sustain a unity of experience. Fourth, as explained before, the dynamical coupling of ZPF modes substantiates the fact that these emergent conscious centers are endowed with structured and variable experiences. Finally, operating in tandem, the structured texture of the emergent phenomenologies, the bifurcation between system and environment, and the fact that there is a great plurality of individuals each conditioning the experiences of others in multiple sorts of ways collectively account for the dual (i.e., subject-object) character of experience. Taken together, we believe that these factors substantiate a minimal sense of perspectival subjectivity.

The above discussion articulates a real sense in which perspectival subjects are grounded as subjects in the aperspectival ground of cosmic consciousness (for an alternative recent account see Albahari forthcoming). It remains to stress that 
beyond all this there is also the issue of the grounding of perspectival selves in the pure self of cosmic consciousness. As mentioned before, we hold that cosmic consciousness is a bearer of pure subjectivity, namely, of a conscious presence devoid of form or objects yet ready to assume particulate qualitative tones and to serve as the apprehending recipient of objects. The emergence of dynamically stable systems in energetic equilibrium with the ZPF has the effect that each of these attractors continually modulates the local field in a particular manner. As a result, each of them appropriates to itself a selective portion of this universal subjective medium, molding it into a private realm engulfed by impulses, experiences, and endeavors-an ego aware of its objects and desires as it is unaware of its substantive unity with other selves and the ground of all being.

In sum, in as much as the problem of the constitution of subjects, as applied to cosmopsychism, is to articulate a substantive and informative ontological sense in which the subjectivity of created subjects is intelligently grounded in the subjectivity of cosmic consciousness, we believe that our account goes a long way toward addressing the challenge. Part of what makes the subject constitution problem so intractable is that it has been shown to repeatedly involve serious conceptual aporia. However, most, if not all, of these conceptual tangles appear to be related to the assumption that one perspectival subject is literally composed of, or fractured from, another (see section I). In the idiom of cosmopsychism, the assumption is that the cosmos itself is a universal mind and that all lesser minds partake in it like colored tiles cut from a jigsaw puzzle's cardboard model-each carrying about itself a small piece of the grand picture. In contrast, our own approach assumes neither the existence of a universal perspectival subject nor a phenomenal decombination thereof. Put differently, our explanation of the emergence of subjects against the background of cosmic consciousness is free from problematic principles of phenomenal combination and perspectival inclusion. We hope we have done enough to elucidate the possibility of this alternative outlook and to motivate further investigations into its ultimate viability.

\section{Implications and Explications}

Having laid down the essentials of our approach, we would like in closing to address a few issues of general import to our enterprise-partly in order to address potential worries and partly with the purpose of pointing to relevant key questions that are beyond the scope of the present work.

One potential concern that might be raised with regard to our account is that the basic theoretical assumptions of our approach fail to close the explanatory gap concerning phenomenal consciousness. Why should the existence of a background field of cosmic consciousness, stirred into specific patterns of phase-locked ZPF modes, yield individual states of phenomenal consciousness? Could we not conceive that all of these activities occur and yet no ordinary states of phenomenal consciousness take place?

In response, we note first that questions of conceivability are hard to settle. To be sure, one can imagine without contradiction that the ZPF is not a carrier of (or identical with) cosmic consciousness or that the formation of phase-locked ZPF 
modes is not the proper mechanism for extracting specific phenomenal states out of pure consciousness. But if one accepts the assumption that there is an underlying field of unlimited phenomenal spectrum and unbounded subjectivity whose default state is that of an undifferentiated unity as well as the assumption that the phase locking of field modes is an appropriate mechanism for differentiating this primal background field into partitioned classes of restricted phenomenal range and bounded subjectivity, is it then still conceivable that all the machinery described in sections 2 and 3 is in place and yet no ordinary phenomenal experiences ensue? Even though we find this question hard to answer beyond dispute, we hold the view that the ocean of consciousness in combination with the mechanism described above necessitates the existence of perspectival subjects endued with phenomenal awareness.

On this note, it is useful to observe the difference between the present scenario and the one that sustains the explanatory gap in the case of materialist theories of consciousness. In the latter case, conceivability arguments reflect the notion that we have principled reasons to doubt that phenomenal facts are necessitated by purely structural (or functional or organizational) facts-no matter what type of structural facts are involved (see Chalmers 20I7). In contrast, on the present scenario the relevant structural facts (concerning the organization of the ZPF via phase locked field modes) are tasked not with the generation of experience per se but, rather, with its modulation and restricted expression, a task description that is well within the capabilities of organization and structure. What constitutes the right kind of organization is, of course, a matter of dispute, but there is no ground for deep skepticism regarding the very notion that some kind of organization sustains the grounding of ordinary experience in fundamental consciousness. In this vein, we motivate our specific account on both philosophical and scientific grounds.

Furthermore, as stressed earlier in sections 2 and 5, the deepest reason for believing the combination problem to be unsolvable in principle is due to the suspicion that intersubjective inclusion relations are downright incoherent (see Coleman [20I4] for an argument to this effect against micropsychism, and Albahari [forthcoming] for an argument against cosmopsychism). This specter of incoherence breeds skepticism regarding the notion that any emergent organization could ever suffice to explain the grounding of ordinary experience in fundamental experience (if nothing could solve the problem, surely no proposed emergent organization could). By demonstrating that the postulate of subjective inclusion is avoidable, our proposal diminishes the case for deep skepticism regarding the prospects for closing the explanatory gap between fundamental experience and ordinary experience-and in doing so it moves the discussion forward.

A second important question relates to the relevance of SED to the formulation of our conceptual framework. In a nutshell, the approach presented is based on the conviction that in order to integrate consciousness coherently into the scientific worldview one has to resort to the most basic level of physics and follow the path of quantum theory. Since SED, as set out in section 2, affords a look behind the scenes of standard quantum theory, it is a natural choice to build our framework on SED. In particular, SED's advantage over standard quantum theory is that it sustains a more informative explanation of the interactive process through which individual 
systems and the background field mutually influence each other. It should then be expected that if phenomenal qualities are dynamically extracted from the background field, as our filtering hypothesis maintains, then it is SED that provides the most informative account of this process. And it does so on a level that cannot be achieved by the apparatus of conventional quantum theory. As a result, new perspectives open up for the development of a fundamental theory of consciousness that preserves the principle of causal closure and respects the law of parsimony, which is reflected in the idea that by use of one and the same mechanism quantum systems acquire both their physical properties and their phenomenal qualities.

Another general question regarding our account pertains to its metaphysical status. The key concept of our proposal-the notion of a cosmic background field-is susceptible of two distinct metaphysical renderings: an idealist interpretation according to which it is strictly a field of consciousness and a double-aspect interpretation that sees it as the carrier of both primordial energy and primordial consciousness. As presented here (and despite some shifting overtones in either direction), our proposal remains agnostic regarding the choice between these two competing alternatives. We take full cognizance of the fact that the question which alternative is to be preferred is substantive: each of these two interpretations presents a different picture of reality, faces unique theoretical challenges, connects with a distinct philosophical pedigree, and has its own champions and detractors. There is no doubt that the question must ultimately be addressed. Nevertheless, we consider it an advantage of our approach that it can be formulated and evaluated in relative independence of the issue of its ultimate metaphysical interpretation.

We offer a novel hypothesis with a coherent research agenda. Our approach can be assessed with respect to its performance along crucial valuation standards, such as (a) addressing issues of mental combination and the emergence of ordinary experience; (b) delineating and explaining systematic connections between ZPF information states and articulated phenomenal states, resulting in the derivation of psychophysical mapping rules between particular qualia and particular sets of phase-locked ZPF modes, thus shedding light on the internal structure of qualia space (see Keppler 20I6); and (c) meeting suitability criteria as a general theoretical framework concordant with robust empirical knowledge concerning the neural correlates of consciousness (see Keppler 20I6, 20I8). All of this, we maintain, can be done independently of settling the question of precise metaphysical interpretation-a question that, given its gravity and the complicated nature of the issues involved, we cannot hope to address properly on the present occasion. Moreover, we think it is reasonable to expect that a better understanding of the manner in which the ZPF grounds ordinary phenomenal states (if indeed it does) could prove itself instrumental in clarifying the nature of the underlying metaphysical landscape.

ITAY SHANI

SUN YAT SEN UNIVERSITY ishani479@hotmail.com 


\section{References}

Albahari, M. (Forthcoming) 'Beyond Cosmopsychism and the Great I Am: How the World might be Grounded in Universal “Advaitic" Consciousness'. In William Seager (ed.), The Routledge Handbook of Panpsychism (New York, NY: Routledge).

Almaas, A. H. (2004) The Inner Journey Home: The Soul's Realization of the Unity of Reality. Boston: Shambhala.

Basile, P. (2010) 'It Must be True - But How Can it Be? Some Remarks on Panpsychism and Mental Composition'. Royal Institute of Philosophy Supplement, 67, 93-I I 2.

Boyer, T. H. (I969) 'Derivation of the Blackbody Radiation Spectrum without Quantum Assumptions'. Physical Review, I 82, I374-83.

Boyer, T. H. (1975) 'Random Electrodynamics: The Theory of Classical Electrodynamics with Classical Electromagnetic Zero-point Radiation'. Physical Review D, I I, 790-808.

Brüntrup, G. (20I7) 'Emergent Panpsychism'. In Godehard Brüntrup and Ludwig Jaskolla (eds.), Panpsychism: Contemporary Perspectives (New York, NY: Oxford University Press), 48-72.

Chalmers, D. J. (I996) The Conscious Mind: Towards a Fundamental Theory. New York: Oxford University Press.

Chalmers, D. J. (20I 5) 'Panpsychism and Panprotopsychism'. In Torin Alter and Yujin Nagawawa (eds.), Consciousness in the Physical World: Essays on Russellian Monism (New York, NY: Oxford University Press), 246-76.

Chalmers, D. J. (20I7) 'The Combination Problem for Panpsychism'. In Godehard Brüntrup and Ludwig Jaskolla (eds.), Panpsychism: Contemporary Perspectives (New York, NY: Oxford University Press), I79-2I4.

Chalmers, D. J. (Forthcoming) 'Idealism and the Mind-Body Problem'. In William Seager (ed.), The Routledge Handbook of Panpsychism (New York, NY: Routledge).

Coleman, S. (2OI2) 'Mental Chemistry: Combination for Panpsychists'. Dialectica, 66, I37-66.

Coleman, S. (20I4) 'The Real Combination Problem: Panpsychism, Micro-subjects, and Emergence'. Erkenntnis, 79, I9-44.

Dainton, B. F. (20II) 'Review of Consciousness and Its Place in Nature'. Philosophy and Phenomenological Research, 83, 238-6I.

De la Peña, L., and A. M. Cetto. (I994) 'Quantum Phenomena and the Zeropoint Radiation Field'. Foundations of Physics, 24, 917-48.

De la Peña, L., and A. M. Cetto. (I995) 'Quantum Phenomena and the Zeropoint Radiation Field II'. Foundations of Physics, 25, 573-604.

De la Peña, L., and A. M. Cetto. (200I) 'Quantum Theory and Linear Stochastic Electrodynamics'. Foundations of Physics, 3I, I703-3 I.

De la Peña, L., and A. M. Cetto. (2006) 'The Foundations of Linear Stochastic Electrodynamics'. Foundations of Physics, 36, 350-68.

De la Peña, L., A. Valdés-Hernández, and A. M. Cetto. (2009) 'Quantum Mechanics as an Emergent Property of Ergodic Systems Embedded in the Zero-point Radiation Field'. Foundations of Physics, 39, I $240-72$.

De la Peña, L., A. M. Cetto, and A. Valdés-Hernández. (2015) The Emerging Quantum: The Physics Behind Quantum Mechanics. Cham: Springer International Publishing.

De la Peña-Auerbach, L., and A. M. Cetto. (I977) 'Derivation of Quantum Mechanics from Stochastic Electrodynamics'. Journal of Mathematical Physics, I8, I6I 2-2 I.

Del Giudice, E., A. De Ninno, M. Fleischmann, G. Mengoli, M. Milani, G. Talpo, and G. Vitiello. (2005) 'Coherent Quantum Electrodynamics in Living Matter'. Electromagnetic Biology and Medicine, 24, 199-2 Iо.

Desmedt, J. E., and C. Tomberg. (1994) 'Transient Phase-locking of $40 \mathrm{~Hz}$ Electrical Oscillations in Prefrontal Parietal Cortex Reflects the Process of Conscious Somatic Perception'. Neuroscience Letters, I68, I26-9.

Engel, A. K., and W. Singer. (200I) 'Temporal Binding and the Neural Correlates of Sensory Awareness'. Trends in Cognitive Sciences, 5, I6-25.

Fasching, W. (20II) 'I Am of the Nature of Seeing: Phenomenological Reflections on the Indian Notion of Witness-consciousness'. In Mark Siderits, Evan Thompson, and Dan Zahavi (eds.), 
Self, No self? Perspectives from Analytical, Phenomenological, and Indian Traditions (Oxford: Oxford University Press), I93-216.

Freeman, W. J. (2007) 'Indirect Biological Measures of Consciousness from Field Studies of Brains as Dynamical Systems'. Neural Networks, 20, I02 I-3 I.

Freeman, W. J., and G. Vitiello. (2007) 'The Dissipative Quantum Model of Brain and Laboratory Observations'. Electronic Journal of Theoretical Physics, 4, I-I 8.

Goff, P. (2006) 'Experiences Don't Sum'. Journal of Consciousness Studies, I3, 53-6I.

Goff, P. (2009) 'Why Panpsychism doesn't Help Us Explain Consciousness'. Dialectica, 63, 2893 II.

Goff, P. (2017) Consciousness and Fundamental Reality. Oxford: Oxford University Press.

Goff, P. (Forthcoming) 'Micropsychism, Cosmopsychism, and the Grounding Relation'. In William Seager (ed.), The Routledge Handbook of Panpsychism (New York, NY: Routledge).

James, W. ([1890] 1950) The Principles of Psychology (vol. I). Mineola, NY: Dover Publications.

Jaskolla, L. J., and A. J. Buck. (20I2) 'Does Panexperiential Holism Solve the Combination Problem?' Journal of Consciousness Studies, I9, 190-9.

Keppler, J. (20I2) 'A Conceptual Framework for Consciousness Based on a Deep Understanding of Matter'. Philosophy Study, 2, 689-703.

Keppler, J. (2013) 'A New Perspective on the Functioning of the Brain and the Mechanisms behind Conscious Processes'. Frontiers in Psychology, 4, 242.

Keppler, J. (20I6) 'On the Universal Mechanism Underlying Conscious Systems and the Foundations for a Theory of Consciousness'. Open Journal of Philosophy, 6, 346-67.

Keppler, J. (20I8) 'The Role of the Brain in Conscious Processes: A New Way of Looking at the Neural Correlates of Consciousness'. Frontiers in Psychology, 9, I346.

Lockwood, M. (1993) 'The Grain Problem'. In Howard Robinson (ed.), Objections to Physicalism (Oxford: Oxford University Press), 27I-9I.

Marshall, T. W. (I963) 'Random Electrodynamics'. Proceedings of the Royal Society of London A, 276, 475-9I.

Marshall, T. W. (1965) 'Statistical Electrodynamics'. Proceedings of the Cambridge Philosophical Society, 6I, 537-46.

Mathews, F. (20II) 'Panpsychism as Paradigm'. In Michael Blamauer (ed.), The Mental as Fundamental: New Perspectives on Panpsychism (Heusenstamm: Ontos Verlag), I4 I-56.

Melloni, L., C. Molina, M. Pena, D. Torres, W. Singer, and E. Rodriguez (2007). 'Synchronization of Neural Activity across Cortical Areas Correlates with Conscious Perception'. Journal of Neuroscience, 27, 2858-65.

Miller, G. (20I8) 'Can Subjects Be Proper Parts of Subjects? The De-Combination Problem'. Ratio, $3 \mathrm{I}, \mathrm{I} 37-54$.

Mørch, H. H. (2014) 'Panpsychism and Causation: A New Argument and a Solution to the Combination Problem'. PhD diss., University of Oslo.

Nagasawa, Y., and K. Wager. (2017) 'Panpsychism and Priority Cosmopsychism'. In Godehard Brüntrup and Ludwig Jaskolla (eds.), Panpsychism: Contemporary Perspectives (New York, NY: Oxford University Press), I I 3-29.

Rodriguez, E., N. George, J. P. Lachaux, J. Martinerie, B. Renault, and F. J. Varela. (I999) 'Perception's Shadow: Long Distance Synchronization of Human Brain Activity. Nature, 397, 430-33.

Roelofs, L. (2015) 'Combining Minds: A Defence of the Possibility of Experiential Combination'. $\mathrm{PhD}$ diss., University of Toronto.

Rosenberg, G. (2004) A Place for Consciousness: Probing the Deep Structure of the Natural World. New York: Oxford University Press.

Seager, W. E. (1995) 'Consciousness, Information, and Panpsychism'. Journal of Consciousness Studies, 2, 272-88.

Seager, W. E. (20I7) 'Panpsychist Infusion'. In Godehard Brüntrup and Ludwig Jaskolla (eds.), Panpsychism: Contemporary Perspectives (New York, NY: Oxford University Press), 229-48.

Sellars, W. (1965) 'The Identity Approach to the Mind-body Problem'. Review of Metaphysics, I 8, $430-5$ I. 
Shani, I. (2015) 'Cosmopsychism: A Holistic Approach to the Metaphysics of Experience'. Philosophical Papers, 44, 389-437.

Smolin, L. (2015) 'Temporal Naturalism'. Studies in History and Philosophy of Science Part B: Studies in History and Philosophy of Modern Physics, 52, 86-102.

Sokolowski, R. (2000) Introduction to Phenomenology. New York, NY: Cambridge University Press.

Strawson, G. (2006) 'Realistic Monism: Why Physicalism Entails Panpsychism'. Journal of Consciousness Studies, I3, 3-3 I.

Strawson, G. (Forthcoming) 'Realistic Idealism, or What Does "Physical” Mean?' In William Seager (ed.), The Routledge Handbook of Panpsychism (New York, NY: Routledge).

Thompson, E. (2015) Waking, Dreaming, Being. New York, NY: Columbia University Press.

Zahavi, D. (2005) Subjectivity and Selfhood: Investigating the First-person Perspective. Cambridge, MA: MIT Press. 